\title{
UNIQUENESS AND NONUNIQUENESS \\ OF THE POSITIVE CAUCHY PROBLEM \\ FOR THE HEAT EQUATION ON RIEMANNIAN MANIFOLDS
}

\author{
MINORU MURATA
}

(Communicated by Peter Li)

\begin{abstract}
We investigate a uniqueness problem of whether a nonnegative solution of the heat equation on a noncompact Riemannian manifold is uniquely determined by its initial data. A sufficient condition for the uniqueness (resp. nonuniqueness) is given in terms of nonintegrability (resp. integrability) at infinity of -1 times a negative function by which the Ricci (resp. sectional) curvature of the manifold is bounded from below (resp. above) at infinity. For a class of manifolds, these sufficient conditions yield a simple criterion for the uniqueness.
\end{abstract}

\section{INTRODUCTION}

Widder [W] established in 1944 that a nonnegative solution of the heat equation on $R^{n}$ is determined uniquely by its initial data. This uniqueness theorem was subsequently extended to parabolic equations on Riemannian manifolds (cf. [Ar], [Az], [AT], [Cha], [D2], [Dod], [Don], [Fri], [KT], [KL], [LY], [MT], $[\mathrm{M} 3,4,5],[\mathrm{N}],[\mathrm{Su}])$. Among others, Karp-Li-Yau ([KL] and [LY]) showed that if Ricci curvatures on a geodesic ball of radius $R$ in a complete Riemannian manifold $M$ are bounded from below by $-C_{1} R^{2}-C_{2}$, where $C_{1}, C_{2}$ are positive constants independent of $R$, then there holds the Widder uniqueness theorem to the heat equation on $M$. On the other hand, Azencott [Az] implicitly showed in his study of conservation of probability that if $M$ is a simply connected analytic complete Riemannian manifold whose sectional curvatures on a geodesic sphere of radius $R$ are bounded from above by $-C R^{2+\epsilon}$ for some positive constants $C$ and $\epsilon$, then the Widder uniqueness theorem to the heat equation on $M$ does not hold.

In this paper we give a necessary and sufficient condition for the Widder uniqueness theorem to the heat equation on a noncompact Riemannian manifold to hold.

Let $M$ be an $n$-dimensional ( $n \geq 2)$, connected, $C^{\infty}$, noncompact, complete Riemannian manifold without boundary. Consider a nonnegative classical

Received by the editors July 2, 1993 and, in revised form, September 13, 1993.

1991 Mathematics Subject Classification. Primary 58G11, 35K05, 35K15; Secondary 58G32.

Key words and phrases. Uniqueness, positive Cauchy problem, heat equation, Riemannian manifold. 
solution of the Cauchy problem

$$
\begin{aligned}
\left(\partial_{t}-\Delta\right) u(x, t) & =0 & & \text { in } \quad M \times(0, T], \\
u(x, 0) & =u_{0}(x) & & \text { on } \quad M,
\end{aligned}
$$

where $\Delta$ is the Laplace-Beltrami operator for $M, \partial_{t}=\partial / \partial t$, and $T$ is a positive constant. We say that UPH (uniqueness of the positive Cauchy problem for the heat equation) holds for $M$ when any two nonnegative solutions $u$ and $\tilde{u}$ of the heat equation (1.1) having the same initial value are identically equal on $M \times[0, T]$; note that no global conditions are imposed on solutions.

The purpose of this paper is to point out how curvatures of a noncompact Riemannian manifold determine whether UPH holds for it or not.

In order to state our main results, Theorems A, B, and C below, we need some more notation. Let $T_{p} M$ be the tangent space to $M$ at a point $p$ in $M$, and $U_{p} M=\left\{\xi \in T_{p} M ;|\xi|=1\right\}$. Let $B_{p}(R)$ be a geodesic ball of radius $R$ centered at $p$ in $M: B_{p}(R)=\{q \in M ; d(p, q)<R\}$. Denote by $K(X, Y)$ the sectional curvature of a plane spanned by linearly independent tangent vectors $X$ and $Y$, and by $\operatorname{Ric}(X)$ the Ricci curvature in the direction $X$.

Theorem A. Suppose that $K(R)$ is a positive continuous increasing function on $[0, \infty)$ such that for any $R>0$

$$
\inf \left\{\operatorname{Ric}(\xi) ; \xi \in U_{q} M, q \in B_{p}(R)\right\} \geq-K(R) .
$$

If

$$
\int_{1}^{\infty} \frac{d r}{\max (\sqrt{K(r)}, r)}=\infty
$$

then UPH holds for $M$.

In the following theorem we shall consider a solution $f$ of the initial value problem

$$
\begin{aligned}
& f^{\prime \prime}=k(r) f \quad \text { in }(0, \infty), \\
& f(0)=0, \quad f^{\prime}(0)=1,
\end{aligned}
$$

where $k$ is a continuous function on $[0, \infty)$.

Theorem B. Assume that the exponential map at a point $p$ is a diffeomorphism from $T_{p} M$ onto $M$. Suppose that $k(R)$ is a continuous increasing function on $[0, \infty)$ such that for any $R>0$

$$
-k(R) \geq \sup \left\{K(X, \dot{\gamma}(R ; q)) ; \quad X \in U_{q} M, q \in \partial B_{p}(R),<X, \dot{\gamma}(R ; q)>=0\right\},
$$

where $\gamma(t ; q)$ stands for the shortest normal geodesic joining $p$ and $q$. Assume that $k\left(R_{0}\right)>0$ for some $R_{0} \geq 0$ and the solution $f$ of $(1.5)-(1.6)$ satisfies

$$
f>0 \text { in }(0, \infty), \quad \lim _{t \rightarrow \infty} f(t)=\infty \text {. }
$$

If

$$
\int_{R_{0}}^{\infty} \frac{d r}{\sqrt{k(r)}}<\infty
$$

then UPH does not hold for $M$. 
The following theorem, which is a direct consequence of Theorems A and B, gives a necessary and sufficient condition for UPH to hold for $M$ belonging to some class of Riemannian manifolds.

Theorem C. Suppose that $M$ is simply connected. Assume that there exist a point $p$ in $M$, positive constants $a, b$, and a positive continuous increasing function $k$ on $[0, \infty)$ such that for any $R>0, q \in \partial B_{p}(R)$, and $X, Y \in U_{q} M$ with $\langle X, Y\rangle=0$, the sectional curvature $K(X, Y)$ satisfies

$$
-b k(R) \leq K(X, Y) \leq-k(R) \leq-a R^{2} .
$$

Then UPH holds for $M$ if and only if

$$
\int_{1}^{\infty} \frac{d r}{\sqrt{k(r)}}=\infty
$$

The rest of this paper is organized as follows. Theorems A and B are proved in Sections 2 and 3, respectively. In Section 4 we shall apply Theorems A and B to a rotationary symmetric Riemannian manifold $M$, and show (see Theorem 4.1) that UPH holds for $M$ if and only if -1 times the radial curvature of $M$ satisfies (1.11). In constructing examples the theorem there yields a useful method. In Section 5 we shall give concluding remarks.

The author thanks Yoshiaki Maeda and Hisao Inoue for helpful conversations.

\section{Proof of Theorem A}

For the proof of Theorem A we make use of a neat uniqueness theorem of Grigor'yan extending that of Täcklind for the heat equation on $R^{n}$ (cf. [T]).

Lemma 2.1 ([G, Theorem 2]). Let $u$ be a solution of (1.1)-(1.2) with $u_{0} \equiv 0$. Assume that for any $R>1$

$$
\int_{0}^{T} d t \int_{B_{p}(R)} u(x, t)^{2} d V(x) \leq C e^{R \rho(R)},
$$

where $\rho(R)$ is an increasing continuous positive function such that

$$
\int_{1}^{\infty} \frac{d r}{\rho(r)}=\infty
$$

Then $u \equiv 0$ on $M \times[0, T]$.

Proof of Theorem A. Let $p(x, y, t)$ be a minimal fundamental solution to the heat equation on $M$ (cf. [Cha]). Then

$$
v(x, t)=\int_{M} p(x, y, t) u_{0}(y) d V(y)
$$

is a solution of (1.1)-(1.2) satisfying $0 \leq v \leq u$ on $M \times[0, T]$. Thus it suffices to show that a nonnegative solution $u$ of $(1.1)-(1.2)$ with $u_{0}=0$ must be identically zero on $M \times[0, T]$. Suppose that $u$ is a nonnegative solution with zero initial data. We see that a function $U(x, t)$ on $M \times[-1, T]$ defined by

$$
U(x, t)= \begin{cases}0 & \text { on } M \times[-1,0], \\ u(x, t) & \text { on } M \times[0, T]\end{cases}
$$


is a nonnegative solution of $(1.1)$ with $(0, T]$ replaced by $(-1, T]$. Thus the proof of Theorems 1.2 and 2.1 of [LY] shows (let the constant $\alpha$ there be 4 ) that there exists a positive constant $C_{1}$ such that

$$
\begin{aligned}
u(x, t) \leq & u(p, t+s)\left(\frac{t+s+1}{t+1}\right)^{2 n} \\
\quad & \quad \exp \left\{\frac{d(p, x)^{2}}{s}+C_{1} s\left[K(2 R)+\frac{1}{R^{2}}+\sqrt{K(2 R)}\right]\right\}, \\
& x \in B_{p}(R), \quad R>1,0 \leq t<t+s \leq T .
\end{aligned}
$$

Put $J(R)=\max \left(K(R), R^{2}\right)$. Since $\sup _{0<\tau<T} u(p, \tau)<\infty$, there exist positive constants $C$ and $m$ such that

$$
u(x, t) \leq C \exp \left(\frac{R^{2}}{s}+m^{2} J(2 R) s\right), \quad x \in B_{p}(R), \quad R>1, \quad 0 \leq t<t+s \leq T .
$$

Fix $\delta$ such that $0<\delta<T$. We claim that

$$
u(x, t) \leq C \exp \left(\left[2 m+\frac{1}{\delta}\right] R \sqrt{J(2 R)}\right), \quad x \in B_{p}(R), \quad R>1, \quad 0 \leq t \leq T-\delta .
$$

When $\delta \geq R / m \sqrt{J(2 R)}$, we get (2.6) by putting $s=R / m \sqrt{J(2 R)}$ in (2.5). When $\delta<R / m \sqrt{J(2 R)}$, we have

$$
\frac{R^{2}}{\delta}+m^{2} J(2 R) \delta \leq \frac{R^{2}}{\delta}+m R \sqrt{J(2 R)} \leq\left(\frac{1}{\delta}+m\right) R \sqrt{J(2 R)} .
$$

Thus we get (2.6) by putting $s=\delta$ in (2.5). This proves the claim. On the other hand, Bishop's volume comparison theorem (cf. [Sa] and [Cha]) yields

$$
\begin{aligned}
\operatorname{vol}\left(B_{p}(R)\right) & \leq \omega_{n-1} \int_{0}^{R}\left(\sqrt{\frac{n-1}{K(R)}} \sinh \sqrt{\frac{K(R)}{n-1}} r\right)^{n-1} d r \\
& \leq C_{2} \exp (\sqrt{n-1} R \sqrt{K(2 R)})
\end{aligned}
$$

for any $R>1$, where $\omega_{n-1}$ is the area of the $(n-1)$-dimensional unit sphere and $C_{2}$ is a positive constant independent of $R$. Combining (2.6) and (2.7), we obtain

$$
\begin{aligned}
& \int_{0}^{T-\delta} d t \int_{B_{p}(R)} u(x, t)^{2} d V(x) \\
& \quad \leq C^{2} C_{2}(T-\delta) \exp \left(\left[4 m+\frac{2}{\delta}+\sqrt{n-1}\right] R \sqrt{J(2 R)}\right)
\end{aligned}
$$

for any $R>1$. This together with (1.4) and Lemma 2.1 shows that $u=0$ on $M \times[0, T-\delta]$; which proves Theorem A, since $\delta$ can be arbitary small. Q.E.D.

\section{Proof of Theorem B}

In this section we prove Theorem B almost along the line given in [M5]. In proving it we make use of a comparison theorem due to Bishop, Rauch, etc. 
(cf. [Cha], [Ka], [Sa]), and exploit a method developed in connection with nonconservation of probability (cf. [Az], [D1], and [Kh]). The proof is divided into several lemmas; among which Lemmas 3.1 and 3.2 below play a technically crucial role.

Let $k(r)$ be the function given in Theorem B, and $f(r)$ the solution of (1.5)-(1.6). Put $F=f^{\prime} / f$.

Lemma 3.1. There exists a positive constant $R>R_{0}$ such that

$$
\begin{aligned}
& \inf _{r>R} F(r)>0, \\
& \int_{R}^{\infty} \frac{d r}{F}<\infty, \\
& \inf _{r>R} \frac{k(r)}{F(r)^{2}}>0 .
\end{aligned}
$$

Proof. We have from (1.5)

$$
F^{\prime}+F^{2}=k \quad \text { in }(0, \infty) .
$$

By (1.8), there exists $R>R_{0}$ such that $f(R)>0$ and $f^{\prime}(R)>0$. Let $g$ be a solution of the initial value problem:

$$
g^{\prime \prime}=k(R) g \quad \text { in } \quad(R, \infty) ; \quad g(R)=f(R), \quad g^{\prime}(R)=f^{\prime}(R) .
$$

With $G=g^{\prime} / g$,

$$
(F-G)^{\prime}+(F+G)(F-G)=k-k(R) \geq 0 \quad \text { in } \quad(R, \infty), \quad(F-G)(R)=0 .
$$

Thus $F \geq G$ on $[R, \infty)$. Since $\lim _{r \rightarrow \infty} G(r)=\sqrt{k(R)}$, this implies (3.1). We next claim that

$$
\frac{1}{F}+\frac{1}{2}\left(\frac{1}{F^{2}}\right)^{\prime} \leq \frac{2}{\sqrt{k}} \quad \text { in } \quad(R, \infty)
$$

By (3.4),

$$
\frac{1}{k} \frac{F^{\prime}}{F^{2}}+\frac{1}{k}=\frac{1}{F^{2}}
$$

When $F^{\prime} \geq 0, \quad F \leq \sqrt{k}$; and so

$$
\frac{1}{F}=F\left(\frac{1}{k}+\frac{1}{k} \frac{F^{\prime}}{F^{2}}\right) \leq \frac{1}{\sqrt{k}}+\frac{F^{\prime}}{F^{3}} .
$$

When $F^{\prime}<0,1 / F \leq 1 / \sqrt{k}$; and so

$$
\frac{1}{F}+\frac{1}{2}\left(\frac{1}{F^{2}}\right)^{\prime}=\frac{1}{F}-\frac{F^{\prime}}{F^{3}}=\frac{2}{F}-\frac{k}{F^{3}}<\frac{2}{\sqrt{k}} \text {. }
$$

This proves the claim. Integrating (3.5) from $R$ to $S$ we have

$$
\int_{R}^{S} \frac{d r}{F}+\frac{1}{2}\left(\frac{1}{F(S)^{2}}-\frac{1}{F(R)^{2}}\right) \leq \int_{R}^{S} \frac{2 d r}{\sqrt{k}}
$$

This together with (3.1) and (1.9) implies (3.2). It remains to prove (3.3). Let $H=F / \sqrt{k}$. Since $k$ is increasing, we have for any $r>s \geq R$,

$$
F(r)-F(s) \geq \sqrt{k(r)}[H(r)-H(s)] .
$$


By (3.4),

$$
F(r)-F(s)=\int_{s}^{r} k(t)\left[1-H(t)^{2}\right] d t .
$$

Thus

$$
H(r)-H(s) \leq \int_{s}^{r} \sqrt{k(t)}\left[1-H(t)^{2}\right] d t .
$$

We claim that

$$
\sup _{r \geq R} H(r) \leq M \equiv \max (1, H(R)) .
$$

On the contrary, suppose that there exists $r>R$ such that $H(r)>M$. Then (3.6) implies

$$
\inf \{s \in[R, r] ; H(t) \geq H(r) \text { for any } t \text { in }[s, r]\}=R .
$$

Thus $H(R) \geq H(r)>H(R)$, which is a contradiction. This proves the claim (3.7), which shows (3.3). Q.E.D.

Consider the initial value problem

$$
\begin{aligned}
& \varphi^{\prime \prime}+(n-1) F \varphi^{\prime}=\varphi \quad \text { in } \quad(R, \infty), \\
& \varphi(R)=1, \quad \varphi^{\prime}(R)=0 .
\end{aligned}
$$

Lemma 3.2. $\varphi, \varphi^{\prime}>0$ in $(R, \infty)$, and $\varphi(\infty) \equiv \lim _{r \rightarrow \infty} \varphi(r)<\infty$.

Proof. The first assertion clearly holds. Put $\Phi=\log \varphi$. By (3.8),

$$
\Phi^{\prime \prime}+(n-1) F \Phi^{\prime}+\left(\Phi^{\prime}\right)^{2}=1 \quad \text { in }(R, \infty) .
$$

This implies

$$
(n-1) \Phi^{\prime} \leq \frac{1}{F}-\frac{\Phi^{\prime \prime}}{F}
$$

We have

$$
-\int_{R}^{r} \frac{\Phi^{\prime \prime}}{F} d s=\frac{\Phi^{\prime}(R)}{F(R)}-\frac{\Phi^{\prime}(r)}{F(r)}+\int_{R}^{r} \Phi^{\prime}\left(\frac{1}{F}\right)^{\prime} d s \leq \frac{\Phi^{\prime}(R)}{F(R)}+\int_{R}^{r} \Phi^{\prime}\left(1-\frac{k}{F^{2}}\right) d s
$$

Thus

$$
\int_{R}^{r} \Phi^{\prime}\left(n-2+\frac{k}{F^{2}}\right) d s \leq \int_{R}^{r} \frac{d s}{F}+\frac{\Phi^{\prime}(R)}{F(R)} .
$$

Hence

$$
\left(\inf _{r>R} \frac{k(r)}{F(r)^{2}}\right)(\Phi(r)-\Phi(R)) \leq \int_{R}^{\infty} \frac{d s}{F}+\frac{\Phi^{\prime}(R)}{F(R)}
$$

for any $r>R$; which shows that $\lim _{r \rightarrow \infty} \varphi(\infty)<\infty$. Q.E.D.

Put

$$
\psi(r)=1-\frac{\varphi(r)}{\varphi(\infty)}, \quad \Psi(x)=\psi(d(p, x))
$$


Lemma 3.3. $\psi$ is decreasing, $0<\psi<1, \lim _{r \rightarrow \infty} \psi(r)=0$, and

$$
(1-\Delta) \Psi \geq 1 \quad \text { in } M \backslash \bar{B}_{p}(R) .
$$

Proof. We have only to prove (3.12). By geodesic spherical coordinates based at $p$,

$$
-\Delta \Psi(x)=\frac{1}{\varphi(\infty)}\left(\varphi^{\prime \prime}(r)+\frac{\partial_{r} \sqrt{\mathbf{g}}}{\sqrt{\mathbf{g}}} \varphi^{\prime}(r)\right),
$$

where $r=d(p, x)$ and $\sqrt{\mathbf{g}}$ is a density function of the area element of $\partial B_{p}(r)$ with respect to the standard area element of the unit sphere $S^{n-1}$ (cf. [Cha, p. 149]). We now apply a comparison theorem (cf. [Ka, Theorem 2.49] and [Sa, Theorem 3.1]) to $\partial_{r} \sqrt{\mathbf{g}} / \sqrt{\mathbf{g}}$, taking as a model manifold a rotationary symmetric Riemannian manifold with radial sectional curvature $-k(r)$ (see [Cho] or Section 4 below), and get

$$
\frac{\partial_{r} \sqrt{\mathbf{g}}}{\sqrt{\mathbf{g}}} \geq(n-1) F,
$$

where $F=f^{\prime} / f$ with $f(r)$ being the solution of (1.5)-(1.6). Since $\varphi^{\prime}>0$, this implies that

$$
(1-\Delta) \Psi(x) \geq 1+\frac{1}{\varphi(\infty)}\left(\varphi^{\prime \prime}(r)+(n-1) F(r) \varphi^{\prime}(r)-\varphi(r)\right)=1 .
$$

Q.E.D.

We are now ready to complete the proof of Theorem B by constructing a positive null solution. Let $p(x, y, t)$ be a minimal fundamental solution for the heat equation on $M$. Put

$$
w(x, t)=\int_{M} p(x, y, t) d V(y) .
$$

Then we see that $w$ is a solution of $(1.1)$ with $w(x, 0)=1$ and $0<w \leq 1$ on $M \times[0, \infty)$.

Lemma 3.4. $0<w<1$ in $M \times(0, \infty)$.

Proof. Put

$$
v(x)=\int_{0}^{\infty} e^{-t} w(x, t) d t .
$$

Then $0<v \leq 1$ and $(1-\Delta) v=1$ on $M$. By Lemma 3.3 and the minimality of $p(x, y, t)$, there exists a positive constant $C$ such that

$$
v(x) \leq C \Psi(x) \quad \text { in } \quad M \backslash \bar{B}_{p}(R)
$$

(cf. [D1, Lemma 2.3]). Thus

$$
\lim _{d(p, x) \rightarrow \infty} v(x)=0,
$$

which implies that $w \neq \equiv 1$. On the other hand, the parabolic Harnack inequality together with the semigroup property of the minimal fundamental solution $p$ implies that either $w \equiv 1$ or $0<w<1$ in $M \times(0, \infty)$. Hence $0<w<1$. Q.E.D. 
Completion of the proof of Theorem B. Put $u(x, t)=1-w(x, t)$. Then we see that $u$ is a solution of $(1.1)$ with $u(x, 0)=0$ and $0<u<1$ in $M \times(0, \infty)$. Q.E.D.

\section{ROTATIONARY SYMMETRIC RIEMANNIAN MANIFOLDS}

Let $M$ be a Riemannian manifold rotationary symmetric at $p$ such that the exponential map at $p$ is a diffeomorphism from $T_{p} M$ onto $M$ (cf. [Cho]). Then the sectional curvature $K(X, \dot{\gamma}(R ; q))$ in (1.7) depends only on $R$, and is called a radial curvature. Denote it by $-k(R)$, and let $f$ be a solution of (1.5)(1.6). Then the Riemannian metric in terms of a geodesic polar coordinates at $p$ is given by

$$
d s^{2}=d r^{2}+f(r)^{2} d \Theta^{2},
$$

where $d \Theta^{2}$ is the standard metric of the unit sphere $S^{n-1}$.

Theorem 4.1. Assume that $k$ is a positive increasing function on $[0, \infty)$ such that $k(r) \geq a r^{2}$ on $[0, \infty)$, where $a$ is a positive constant. Then UPH holds for $M$ if and only if (1.11) holds.

In view of Theorems A and B, Theorem 4.1 is derived from the following lemma.

Lemma 4.2. There exists a positive constant $C$ such that

$$
K(X, Y) \geq-C k(R)
$$

for any $R>0$ and $X, Y \in U_{q} M$ with $\langle X, Y\rangle=0$ and $q \in \partial B_{p}(R)$.

Proof. With $X=(x, v), Y=(0, w)$, where $x \in[-1,1]$ and $v, w \in$ $T_{\theta} S^{n-1}(q=(R, \theta))$, the sectional curvature $K(X, Y)$ is given by

$$
K(X, Y)=\frac{1-f^{\prime}(R)^{2}}{f(R)^{2}}\left(1-x^{2}\right)-\frac{f^{\prime \prime}(R)}{f(R)} x^{2}
$$

(cf. [BO, the formula on p. 27]). Thus, in view of (1.5), it suffices to estimate the function

$$
g(R) \equiv \frac{1-f^{\prime}(R)^{2}}{f(R)^{2}}
$$

Clearly, $\lim _{R \rightarrow 0} g(R)=-k(0)$ and $f, f^{\prime}>0$ in $(0, \infty)$. By (3.3) of Lemma 3.1 , there exists a positive constant $m>0$ such that $k(R) \geq m\left[f^{\prime}(R) / f(R)\right]^{2}$ for any $R>1$. Hence there exists a positive constant $C$ such that $g(R) \geq$ $-C k(R)$ for any $R>0$. This proves the lemma. Q.E.D.

\section{REMARKS}

5.1. When UPH does not hold for $M$, an interesting problem is to determine the structure of all positive solutions $u$ of (1.1)-(1.2) with $u_{0}=0$ and $u>0$ in $M \times(0, T]$. This problem is closely related to a parabolic Martin boundary and Martin kernel (cf. [Fre], [MT], [M3,4], [P]).

5.2. It is of some interest to compare Theorem 4.1 with a delicate criterion on existence of a nonconstant positive harmonic function, which is unstable under constant multiplication of the radial curvature (cf. [M2,1]). 


\section{REFERENCES}

[Ar] D. G. Aronson, Non-negative solutions of linear parabolic equations, Ann. Scuola Norm. Sup. Pisa Cl. Sci. (4) 22 (1968), 607-694.

[AT] A. Ancona and J. C. Taylor, Some remarks on Widder's theorem and uniqueness of isolated singularities for parabolic equations, Partial Differential Equations with Minimal Smoothness and Applications (B. Dahlberg et al., eds.), Springer-Verlag, New York, 1992, pp. 15-23.

[Az] R. Azencott, Behavior of diffusion semi-groups at infinity, Bull. Soc. Math. France 102 (1974), 193-240.

[BO] R. L. Bishop and B. O'Neill, Manifolds of negative curvature, Trans. Amer. Math. Soc. 145 (1969), 1-49.

[Cha] I. Chavel, Eigenvalues in Riemannian geometry, Academic Press, London, 1984.

[Cho] H. I. Choi, Asymptotic Dirichlet problems for harmonic functions on Riemannian manifolds, Trans. Amer. Math. Soc. 281 (1984), 691-716.

[D1] E. B. Davies, $L^{1}$ properties of second order elliptic operators, Bull. London Math. Soc. 17 (1985), 417-436.

[D2] _ Heat kernels and spectral theory, Cambridge Univ. Press, Cambridge, 1989.

[Dod] J. Dodziuk, Maximum principle for parabolic inequalitites and the heat flow on open manifolds, Indiana Univ. Math. J. 32 (1983), 703-716.

[Don] H. Donnelly, Uniqueness of the positive solutions of the heat equation, Proc. Amer. Math. Soc. 99 (1987), 353-356.

[Fre] A. Freire, On the Martin boundary of Riemannian products, J. Differential Geom. 33 (1991), 215-232.

[Fri] A. Friedman, Partial differential equations of parabolic type, Prentice-Hall, Englewood Cliffs, NJ, 1964.

[G] A. A. Grigor'yan, On stochastically complete manifolds, Soviet Math. Dokl. 34 (1987), 310-313.

[Ka] A. Kasue, $A$ Laplacian comparison theorem and function theoretic properties of a complete Riemannian manifold, Japan. J. Math. (N.S.) 8 (1982), 309-341.

[Kh] R. Z. Khas'minskii, Ergodic properties of recurrent diffusion processes and stabilization of the solution to the Cauchy problem for parabolic equations, Theory Probab. Appl. 5 (1960), 179-196.

[KL] L. Karp and P. Li, The heat equation on complete Riemannian manifolds, unpublished.

[KT] A. Koranyi and J. C. Taylor, Minimal solutions of the heat equation and uniqueness of the positive Cauchy problem on homogeneous spaces, Proc. Amer. Math. Soc. 94 (1985), 273-278.

[LY] P. Li and S. T. Yau, On the parabolic kernel of the Schrödinger operator, Acta Math. 156 (1986), 153-201.

[MT] B. Mair and J. C. Taylor, Integral representation of positive solutions of the heat equation, Théorie du Potentiel (G. Mokobodzki and D. Pinchon, eds.), Lecture Notes in Math., vol. 1096, Springer-Verlag, Berlin, Heidelberg, and New York, 1984, pp. 419-433.

[M1] M. Murata, On construction of Martin boundaries for second order elliptic equations, Publ. Res. Inst. Math. Sci. 26 (1990), 585-627.

[M2] , Positive harmonic functions on rotationary symmetric Riemannian manifolds, Potential Theory (M. Kishi, ed.), Walter de Gruyter \& Co., Berlin and New York, 1992, pp. 251-259.

[M3] - Uniform restricted parabolic Harnack inequality, separation principle, and ultracontractivity for parabolic equations, Functional Analysis and Related Topics, 1991 (H. Komatsu, ed.), Lecture Notes in Math., vol. 1540, Springer-Verlag, Berlin, Heidelberg, and New York, 1993, pp. 277-288.

[M4] _ Non-uniqueness of the positive Cauchy problem for parabolic equations, J. Differential Equations (to appear). 
[M5] _ Sufficient condition for non-uniqueness of the positive Cauchy problem for parabolic equations, Spectral and Scattering Theory and Applications (K. Yajima, ed.), Adv. Stud. Pure Math., vol. 23, Kinokuniya, Tokyo, 1994, pp. 275-282.

[N] T. Nagasawa, Uniqueness and Widder's theorems for the heat equation on Riemannian manifolds, Geometry and Its Applications (T. Nagano et al., eds.), World Scientific, Singapore, 1993, pp. 161-173.

[P] Y. Pinchover, Representation theorems for positive solutions of parabolic equations, Proc. Amer. Math. Soc. 104 (1988), 507-515.

[Sa] T. Sakai, Riemannian geometry, Shokabo, Tokyo, 1992. (Japanese)

[Su] N. Suzuki, Huygens property of parabolic functions and a uniqueness theorem, Hiroshima Math. J. 19 (1989), 355-361.

[T] S. Täcklind, Sur les classes quasianalytiques des solutions des équations aux dérivées partielles du type parabolique, Nova Acta Regiae Soc. Sci. Upsaliensis Ser. IV 10 (1936), 1-57.

[W] D. V. Widder, Positive temperatures on an infinite rod, Trans. Amer. Math. Soc. 55 (1944), 85-95.

Department of Mathematics, Faculty of Science, Kumamoto University, Kumamoto, 860 JAPAN

Current address: Department of Mathematics, Tokyo Institute of Technology, Oh-okayama, Meguro-ku, Tokyo, 152 Japan

E-mail address: minoru30math.titech.ac.jp 